Anales de Geografía de la Universidad Complutense ISSN: 0211-9803

http://dx.doi.org/10.5209/AGUC.60471

\title{
Metabolismo insular: flujos y retos del desarrollo territorial en las Islas Galápagos (Ecuador)
}

\author{
Gabriela Rodríguez Jàcome ${ }^{1}$; Asunción Blanco-Romero ${ }^{2}$ \\ Recibido: 15 de diciembre del 2016 / Enviado a evaluar: 1 de junio del 2017 / Aceptado: 14 de diciembre del 2017
}

Resumen. Los espacios insulares son territorios cuyo tamaño, disponibilidad de recursos y accesibilidad son limitados. La insularidad marca unas dinámicas territoriales singulares en estos espacios, que a pesar de su gran valor estratégico los hacen dependientes de las relaciones con el exterior. Las islas Galápagos, pertenecientes a la República del Ecuador, constituyen un caso muy particular de estudio. Si bien las categorías de Patrimonio Natural Mundial y Parque Nacional le dan condiciones de protección de la naturaleza, únicas en el mundo, sus habitantes soportan la misma, o incluso mayor, problemática asociada a la insularidad e isleidad que afrontan otras islas. El presente estudio analiza las relaciones de dependencia de los flujos de turismo, abastos y combustibles entre las islas Galápagos con el Ecuador continental desde la perspectiva del metabolismo insular y la necesidad de adaptación de las políticas y planificación territorial a este contexto. Con este marco este estudio plantea: a) Entender cuáles son los retos del desarrollo territorial considerando el metabolismo insular, b) Considerar la planificación territorial como un aliado de la conservación ambiental, y c) Entender los flujos estratégicos como un factor para reducir la vulnerabilidad y promover la viabilidad insular.

Palabras clave: Insularidad; viabilidad insular; desarrollo territorial; metabolismo insular; Galápagos Ecuador.

\section{[en] Insular metabolism: flows and challenges of territorial development in the Galapagos Islands (Ecuador)}

\begin{abstract}
Island are territories whose size, availability of resources and accessibility are limited. Insularity determines a unique territorial dynamic in these spaces which, despite their great strategic value, make them dependent on relations with the outside world. The Galapagos Islands, belonging to the Republic of Ecuador, constitute a very special case of study. Although the categories of World Natural Heritage and National Park give conditions of protection of nature, unique in the world, its inhabitants endure the same or a greater problem associated with the insularity and isleity that other
\end{abstract}

1 Departamento de Geografía. Universitat Autònoma de Barcelona.

E-mail: ga.diylab@gmail.com

2 Departamento de Geografía. Universitat Autònoma de Barcelona.

E-mail: asuncion.blanco@uab.cat 
islands face. The present study analyzes the dependency relationships of the flows of tourism, supplies and fuels between the Galapagos Islands and mainland Ecuador from the perspective of insular metabolism and the need to adapt policies and territorial planning to this context. With this framework, this study proposes: a) Understanding the challenges of territorial development considering the insular metabolism, b) Considering territorial planning as an ally of environmental conservation, and c) Understanding strategic flows as a factor to reduce vulnerability and promote the insular viability.

Key words: Insularity; insular viability; territorial development; insular metabolism; Galápagos Ecuador.

\section{[fr] Métabolisme insulaire: flux et défis du développement territorial dans les Iles Galapagos (Équateur)}

Résumé. Les espaces insulaires sont des territoires dont la taille, la disponibilité des ressources et l'accessibilité sont limitées. L'insularité marque une dynamique territoriale singulière dans ces espaces, qui en dépit de leur grande valeur stratégique les rendent dépendants des relations avec l'extérieur. Les îles Galapagos, appartenant à la République de l'Equateur, constituent un cas d'étude très particulier. Bien que les catégories du Patrimoine Naturel Mondial et du Parc National offrent des conditions de protection de la nature, uniques au monde, ses habitants subissent une plus grande problématique associée à l'insularité que connaissent les autres îles. La présente recherche analyse les rapports de dépendance des flux du tourisme, des fournitures et des combustibles entre les îles Galapagos et la partie continentale de l'Equateur du point de vue du métabolisme insulaire et la nécessité d'adapter les politiques et la planification territoriale à ce contexte. Dans ce cadre, cette étude propose: a) de comprendre les enjeux du développement territorial en considérant le métabolisme insulaire, b) de considérer la planification territoriale comme un allié de la préservation de l'environnement, et c) de comprendre les flux stratégiques comme facteur de vulnérabilité et promouvoir la viabilité insulaire.

Mots clés: Insularité ; viabilité insulaire; développement territorial; métabolisme insulaire; Galápagos Equateur.

Cómo citar. Rodríguez Jàcome, G. y Blanco-Romero, A. (2018):Metabolismo insular: flujos y retos del desarrollo territorial en las Islas Galápagos (Ecuador). Anales de Geografia de la Universidad Complutense, 38(1), 113-135.

Sumario. 1. Introducción. 1.1. Partiendo de la insularidad o la isleidad. 1.2. El sistema socioecológico y el metabolismo insular. 3. Territorio, uso y dinámicas en Galápagos. 3.1. El contexto territorial. 3.2. Ordenamiento territorial y gestión del área protegida. 3.3. Dinámica poblacional y turística. 4. Flujos estratégicos e insularidad. 4.1. Flujos estratégicos hacia las Islas Galápagos. 4.2. Movilización y transporte entre el Ecuador continental e insular. 4.3. Problemática para determinar el metabolismo insular. 5. Más allá de los datos: impactos y consecuencias de la dependencia. 5.1. Consideraciones con relación a la actividad turística. 5.2. Consideraciones respecto a los combustibles, contaminación ambiental y cambio climático. 5.3. Consideraciones en relación a los abastos. 5.4. Incentivos económicos y políticas contradictorias en las islas Galápagos. 6. A modo de conclusiones. 7. Bibliografía.

\section{Introducción}

Los procesos que se desarrollan tras la dinámica poblacional residencial y turística en las islas Galápagos en relación con las estrategias de abastecimiento de productos y combustibles han sido en general poco estudiados, encontrándose implicados en dichas estrategias múltiples factores y niveles de complejidad. Así, mientras la 
producción local de alimentos, o de autoabastecimiento, están limitados por el contexto de prioridad de conservación del patrimonio natural y las restricciones en la introducción de especies, las demandas de las islas siguen en aumento. Del mismo modo, el consumo de combustibles se incrementa, debido a la necesidad de generación de energía eléctrica y la demanda de transportes para el mantenimiento de actividades turísticas en el territorio.

Para solventar estas necesidades, se generan nuevos flujos de migración de mano de obra continente-islas (Ecuador continental-Islas Galápagos), para el mantenimiento y desarrollo de la agricultura y el actual auge de la construcción. Aumentando aún más la problemática de la conservación de esta área protegida, patrimonio natural de la humanidad. Entonces, el desarrollo sigue el constante bucle que confronta dos realidades: en primer lugar, la realidad del metabolismo insular creciente que exige flujos de ingreso y retorno para mantener la dinámica territorial insular. Versus una escasa y cortoplacista planificación territorial, que no aborda las demandas locales y además entra en conflicto con la conservación, ya que sus objetivos son "aparentemente" opuestos.

En este marco las islas Galápagos requieren que sus políticas de desarrollo territorial se constituyan en primer lugar, a partir del abordaje conceptual de 'territorio complejo' afectado por la insularidad, y tras las dinámicas del metabolismo social que permitan un abordaje integral como socio-ecosistema insular.

\subsection{Partiendo de la insularidad o la isleidad}

El concepto de insularidad o isleidad han sido utilizados de manera indistinta haciendo referencia a las islas, llegando, en algunos momentos, a contradecirse (Pons, 2016) al ser un término controvertido (Baldacchino, 2004). El concepto insularidad se ha convertido en el más extendido dado su tradición y antigüedad, pero cargado de connotaciones negativas, relacionadas directamente con la idea de aislamiento. Según Boadas (2011:148) "La insularidad es un hecho característico de las islas, que se fundamenta en el aislamiento y su discontinuidad geográfica. La separación territorial y las relaciones externas son factores de la insularidad, cuyas magnitudes le dan significado. La insularidad se afirma y se hace más notoria cuando la distancia entre las islas y los territorios continentales es grande e incide en las comunicaciones con el exterior". Así, más allá del concepto insularidad, el caso de Galápagos se ajusta mucho mejor al de isleidad, término acuñado por Moles en 1982, quien la define como una función fenomenológica de las islas a partir de tres dimensiones: la magnitud de la isla, la distancia a las tierras continentales y su carácter microcósmico que le confiere la cantidad de variedad que contiene (Barceló, 1997; Pons, 2016). De este modo, un concepto más global incluye tanto connotaciones negativas como positivas, de apertura o aislamiento respecto del exterior, en relación a fenómenos físicos o humanos (Pons, 2016) y se convierte en una variable que no determina, sino que perfila y condiciona fenómenos físicos y sociales en sus diferentes formas (Baldacchino, 2004), ayudando a estudiar el carácter distintivo de las islas (Hepburn, 2010). 
Por otro lado, es necesario tener en cuenta que el aislamiento puede catalogarse en varios tipos: social, cultural, político, económico. En este caso de estudio, el grado de aislamiento es geográfico y será definido como la carencia de contacto físico o comunicacional, la separación territorial y de relaciones con el exterior, tanto en referencia entre islas como con el continente. Por ello, el aislamiento puede convertirse en un factor de vulnerabilidad si no se garantiza una adecuada calidad de vida, se aseguran los suministros requeridos por los asentamientos humanos a lo que habría que añadir la propensión a sufrir los efectos de los riesgos naturales (Ver Figura 1).

Figura 1. Relaciones entre insularidad, metabolismo social, vulnerabilidad y viabilidad insular.

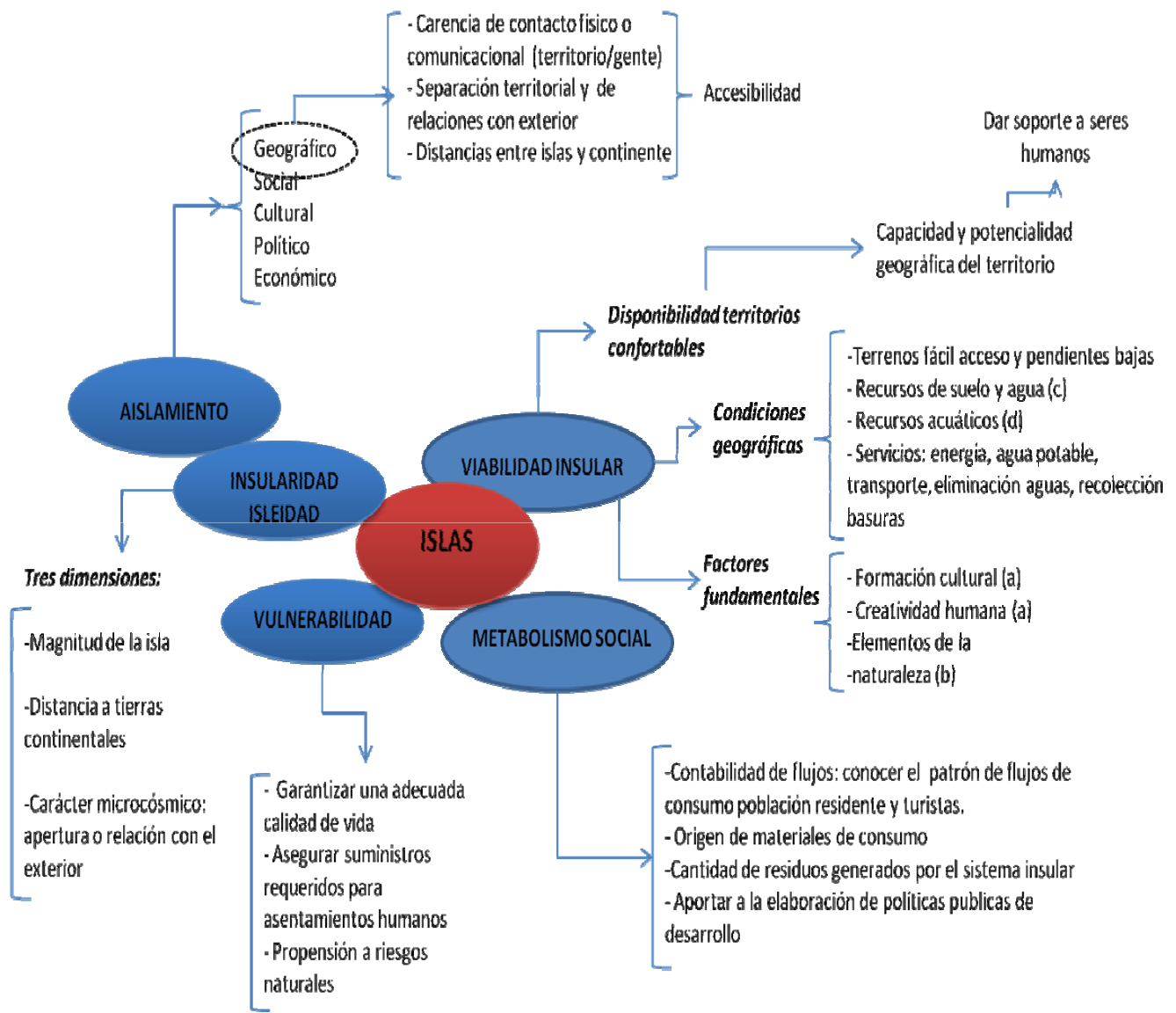

Fuente: Elaboración propia, en base a Hercowitz \& Alier, 2003; Manera \& Garau, 2005; Boadas, 2011 y Rodríguez, 2014. 
En este punto, Galápagos se podría decir que se encuentra plenamente en la metáfora del "metabolismo social", concepto que a partir de diferentes enfoques académicos: la ecología industrial (EI), la economía ecológica (EE), la ecología política y la teoría de la ecología de sistemas (Newell y Cousins, 2014), se centra en la dialéctica socio-ecológica, en términos de los flujos energéticos y materiales necesarios para mantener la estructura interna de los sistemas sociales (FisherKowalski y Haberl 2007). Gracias al análisis de los materiales, bióticos y abióticos, que pasan a través de los sistemas sociales (Eurostat, 2012, Fischer-Kowalski et al., 2011), así como de la llamada Huella Ecológica propuesta por Wackernagel y Rees (1996), la Contabilidad de Flujo de Materiales (MFA) se convierte en una de las principales metodologías utilizadas para conceptualizar el metabolismo social (Murray, 2015).

Con el enfoque del metabolismo social, las islas son considerados organismos vivos, en donde la contabilidad de los flujos materiales permite conocer el patrón de consumo de la población de turistas y residentes, el origen de los materiales que satisfacen el consumo, la cantidad de residuos generados por el sistema insular, con el afán de aportar en la elaboración de políticas públicas para el desarrollo (Hercowitz \& Alier, 2003).

\subsection{El sistema socioecológico y el metabolismo insular}

El concepto metabolismo social entonces es aplicable al estudio 'un metabolismo-una isla', en la cual cada isla debería velar por su integridad. Sin embargo ¿Qué pasa en el caso de los archipiélagos? este conjunto de islas permite la generación de nuevas dinámicas internas (inter-islas) que seguramente reducen la dependencia y vulnerabilidad con el exterior. Desde esta perspectiva, este estudio el metabolismo insular, aborda al archipiélago como un sistema de islas, influido por la insularidadisleidad y cuyo metabolismo social condiciona su funcionamiento. Considerando que cada isla funciona independientemente, en relación a su nivel de insularidad (Manera \& Garau, 2005) y los flujos externos abastecen a todo el sistema insular y se redistribuyen internamente de acuerdo a sus necesidades o accesibilidad, generando nuevos flujos de consumo-desechos al interior. Con ello, las dinámicas inter-islas pueden satisfacer nuevos requerimientos del sistema insular y regular desequilibrios de internos.

Las islas han sido mayoritariamente vulnerables porque generalmente no garantizan los suministros básicos requeridos en los asentamientos humanos, lo que Boadas (2011:149) denomina viabilidad insular. En este caso de estudio, las dinámicas entre el exterior y las islas son denominas flujos estratégicos $\mathrm{y}$, bajo este criterio, se establecen mayores o menores niveles de vulnerabilidad y por tanto de viabilidad insular como objeto de desarrollo.

Mencionar que otros archipiélagos o islas en el mundo están muy desarrollados principalmente en el continente europeo (islas, países insulares, territorios de ultramar) por lo que su viabilidad insular es efectiva y la vulnerabilidad mínima. Sin embargo, no sucede lo mismo en otros pequeños archipiélagos "menos desarrollados" o "más 
protegidos" que podrían ser abordados desde la perspectiva territorial que Capel (2014) denomina espacios no convencionales. O de manera más puntual, desde la antropología ecológica la perspectiva de sistemas complejos adaptativos o socioecosistemas para un análisis más integrador de realidades socio-naturales (Escalera \& Ruiz, 2011). Con ello además se incorpora el término de resiliencia socio-ecológica (Escalera \& Ruiz, 2011) para el estudio de las dinámicas del sistema insular complejo.

En el caso de Galápagos, un archipiélago dependiente del continente, conocido por su naturaleza, con un $97 \%$ de territorio protegido para la conservación estricta (inhabitado) y en donde la legislación se ha enfocado en la conservación como forma de desarrollo, merece la pena ser analizado. Además, considerando, sus categorías de conservación como reserva de la biosfera y patrimonio natural de la humanidad, etiquetas que limitan a su vez su relación con el continente.

En el año 2008 Watkins ya planteó la necesidad de que el archipiélago de Galápagos sea considerado como un sistema socioecológico en donde los sistemas naturales y humanos estén asociados. Sin embargo, su gestión siempre fue y aún apunta a que todo lo proveniente del exterior afecta la conservación del ecosistema insular. Este territorio entonces ha vivido constantemente, con la dinámica diaria de "evitar" al máximo los flujos desde el exterior, pero al mismo tiempo albergar a más de 25 mil habitantes y 200 mil turistas. Este dilema entre "conservación-desarrollo" ha hecho que se limite al máximo el desarrollo territorial considerándolo negativo y tan solo desde el punto de vista urbanizador de la planificación, asumido como un enemigo de los ecosistemas insulares. Con lo cual, se resta la versión de integralidad de la planificación para garantizar un manejo sostenible del socio-ecosistema. Entonces, tras casi 20 años de política de planificación territorial los problemas solo se agravan en la medida que el metabolismo insular no ha sido considerado. Aumentando su nivel de vulnerabilidad y reduciendo su viabilidad insular.

\section{Territorio, uso y dinámicas en las Galápagos}

\subsection{El contexto territorial}

Para situarse en el contexto territorial propio de las Galápagos es necesario tener presente que el archipiélago de las islas Galápagos constituye una de las 24 provincias de la República del Ecuador, se localiza a $960 \mathrm{Km}$ de distancia del Ecuador continental y está conformada por 234 unidades terrestres emergidas (islas, islotes y rocas). Existen 13 islas consideradas grandes cuya superficie es superior a diez kilómetros cuadrado y 216 islotes o promontorios rocosos (DPNG, 2014).

Política y administrativamente las islas Galápagos corresponden a la provincia del mismo nombre, en donde el $97 \%$ de superficie lo constituye el área del Parque Nacional Galápagos (PN) y tan solo el 3\% de la superficie es considerada área de asentamientos humanos (DPNG, 2014).

Con cuatro islas habitadas: San Cristóbal, Santa Cruz, Isabela y Floreana correspondientes a tres cantones y ocho parroquias, tres urbanas y cinco rurales. El 
Archipiélago de Galápagos es una de las cincuenta áreas protegidas de la República del Ecuador. Fue declarada área natural protegida (1959), Patrimonio Natural de la Humanidad (1978), Reserva de la Biosfera (1985) y Santuario Marino (2016).

En las Islas Galápagos el 87,2\% de las actividades están relacionadas con el turismo, lo que motiva una necesidad de planificación y gestión del territorio y sus actividades considerando las dinámicas socioeconómicas locales para garantizar su viabilidad. Como menciona Boadas (2011:147) la viabilidad insular exige la oferta de elementos para satisfacer necesidades humanas básicas y para realizar actividades que contribuyan al mejoramiento de la vida en la isla, además debe fluir una cantidad suficiente y necesaria de energía, nutrientes e información dentro del ecosistema insular.

\subsection{Ordenamiento territorial y gestión del área protegida}

La Ley Orgánica de Régimen Especial de Galápagos (LOREG) ${ }^{3}$ marca que el Consejo de Régimen Especial Gobierno Galápagos (CREGG) es el ente encargado de la planificación, el manejo de los recursos, la organización de las actividades que se realizan en el territorio de la provincia de Galápagos y la coordinación interinstitucional con las instituciones del Estado, en el ámbito de sus competencias. Para la gestión del área protegida dentro de la provincia de Galápagos, la DPNG es la entidad que tiene competencia sobre el uso, manejo y aprovechamiento de los recursos naturales. Las actividades que en dichas áreas se realicen estarán en conformidad con el Sistema Nacional de Áreas Protegidas, en concordancia con el Plan para el Desarrollo Sustentable y Ordenamiento Territorial de Galápagos y las políticas generales de planificación dictadas por el Consejo de Gobierno del Régimen Especial de la provincia de Galápagos.

Con anterioridad a la LOREG (2015) el área terrestre de la ecoregión Galápagos era el espacio donde se realizaba una zonificación para el ordenamiento territorial y gestión del Parque Nacional Galápagos. Por lo tanto, todo el territorio insular estaba incluido bajo la normativa de una zonificación, que para la DPNG (2005) era la expresión espacial de la política de conservación del Parque Nacional Galápagos.

A pesar de albergar espacios de asentamientos humanos, el plan de manejo fue la herramienta con la cual se planificaba el territorio hasta el año 2008 (cuando se crea el CREGG), esto explica cómo con anterioridad los procesos de desarrollo se gestionaban con un marcado sesgo ambiental.

Con ello la importancia del abordaje socio-ecológico como mencionan Escalera \& Ruiz (2011) "Difícilmente una intervención ambiental alcanzará sus objetivos si no se tienen en cuenta las dimensiones biofísicas y socioculturales simultáneamente y con la misma profundidad, estableciendo estrategias y mecanismos que lo hagan factible."

\footnotetext{
${ }^{3}$ Registro Oficial Suplemento 50 de 11-jun.-2015.
} 


\subsection{Dinámica poblacional y turísctica}

Según el Instituto Nacional de Estadísticas y Censos, INEC (2015) la población de las islas Galápagos corresponde a 25.244 habitantes distribuidas geográficamente de la siguiente manera: en el cantón Santa Cruz con 15.701 habitantes, el cantón San Cristóbal con 7199 habitantes y el cantón Isabela con 2.344 habitantes. La isla Floreana perteneciente al cantón San Cristóbal alberga a 111 habitantes.

A diferencia de otros espacios, en Galápagos se aprecia que la población urbana sufre un decrecimiento en comparación con los censos anteriores. Para el 2001 la población urbana era del $85,1 \%$, en el año 2010 de un 83,1\% y en el año 2015 de un 81,4\%. Según el INEC (2015) en el período intercensal 1950-2015 la población de la provincia se ha multiplicado en 18,8 veces.

Con respecto al tema turístico, Ecuador en el año 2015 recibió 1.364 .057 de visitantes, de los cuales casi el $10 \%$ visitaron el archipiélago de Galápagos, constituyéndose así en uno de los principales destinos turísticos del país. Según la evolución del número de turistas que han ingresado en Galápagos desde el año 1979 al 2015 se aprecia un incremento que va desde los 11.765 turistas de 1979 a los 224.755 de 2015 (ver Figura 2).

Figura 2. Número de turistas llegados a Galápagos (1979-2016).

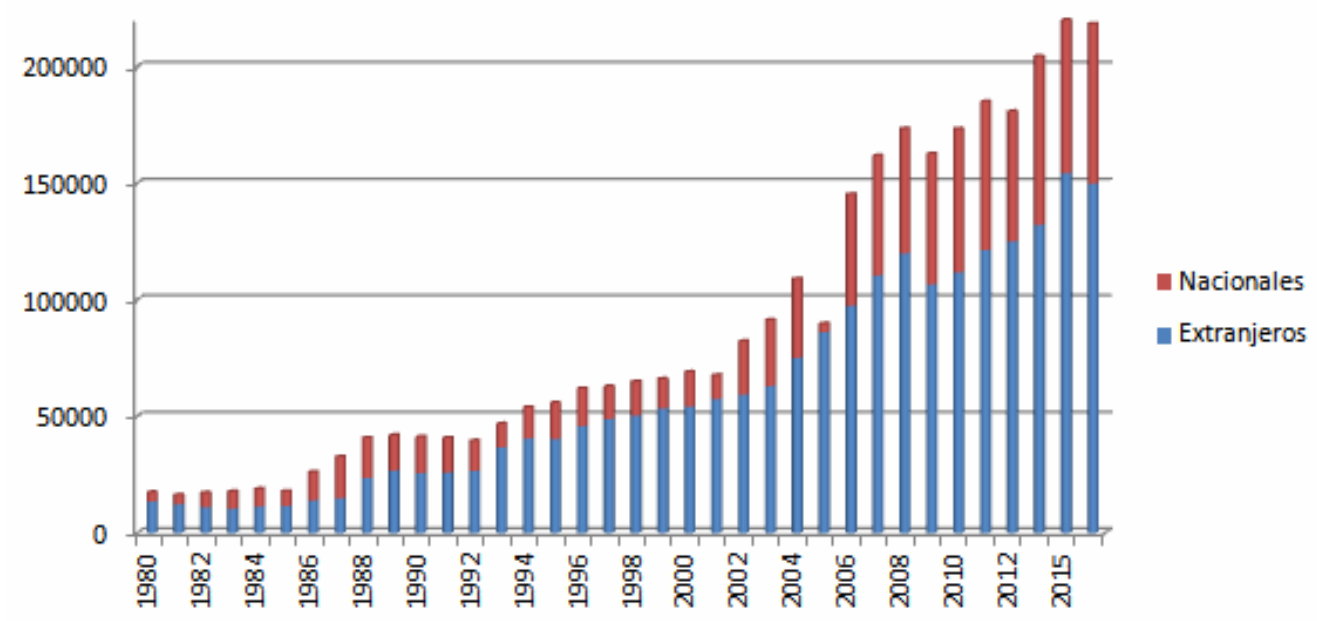

Fuente: Elaboración propia en base a datos de la DPNG, 2017.

Es posible ver como en los años 2003 y 2004, el número de turistas sufre un incremento mayor en comparación a los anteriores años, pasando de 91.356 a 108.948 turistas. Este 19\% de incremento está compuesto, en mayor medida, por visitantes extranjeros que nacionales ecuatorianos. Este crecimiento se ha ido moderando y equilibrando, produciéndose para el año 2015 un aumento del 4\% en las llegadas 
respecto al 2014 y en mayor grado debido al aumento de los turistas nacionales $(+7 \%)$ que para los extranjeros (+3\%). De manera que para el año 2015, el 69\% (154.304) de los visitantes eran extranjeros y el 31\% (70.451) eran nacionales (Ver Figura 2).

Según el tipo de hospedaje utilizado por los visitantes en el año 2015, el $68 \%$ del total de los visitantes que recibió Galápagos utilizó los servicios de alojamiento en tierra, mientras que el $352 \%$ se hospedó a bordo de embarcaciones de crucero o tour navegables (Observatorio de Turismo, 2017).

El ingreso económico por el turismo en Galápagos representaba, según datos de 2014, unos 258 millones de dólares americanos. Según lugares de procedencia de los turistas de las islas Galápagos la distribución, en 2014, muestra el predominio de los turistas nacionales ecuatorianos con un $30 \%$, seguidos de los procedentes de Estados Unidos con un 26\%, Reino Unido un 6\%, Alemania, Canadá y Australia un $4 \%$ cada uno, Argentina un $3 \%$ y finalmente otros países el $23 \%$.

En cuanto a experiencia turística en Galápagos el turismo es principalmente de aventura y de naturaleza. A nivel del archipiélago existen 135 sitios de visita, de los cuales 75 son lugares de visita marinos y 70 terrestres. Según Pizzitutti et al. (2014) las experiencias de turismo en Galápagos se dividen en dos segmentos principales: el turismo terrestre y el turismo basado en la embarcación. Así, los turistas que reservan un crucero para explorar las islas Galápagos se definen como "basado en barco" porque, en general, pasan la mayor parte del tiempo de su experiencia turística a bordo -para dormir, comer-, y un corto desembarque para actividades en las islas. Este tipo de turistas es percibido por los residentes como "el que no contribuye a la economía local" (Epler, 2007), ya que los fondos tienden a revertir en las empresas nacionales e internacionales de las embarcaciones y en las organizaciones de turismo de gran tamaño. La experiencia turística en tierra, en cambio, se basa en las comunidades locales, organizando las actividades turísticas con los proveedores locales, comiendo en sus hoteles o en restaurantes locales, etc. Estos turistas son percibidos por la población local como más dependientes y relacionados directamente con los productos locales, los trabajadores y los servicios de la zona (Pizzitutti et al. 2014).

\section{Flujos estratégicos e insularidad}

La insularidad de dimensiones reducidas ha implicado en Galápagos, sobre todo, una limitada capacidad de carga, tanto natural como económica y social. Los ecosistemas insulares, o cada isla como un socio-ecosistema cerrado a una determinada escala, su limitada dotación en recursos naturales y humanos hacen de las islas espacios especialmente frágiles y poco aptos para acoger actividades con un elevado consumo de medios de producción y de territorio, sin riesgo de que se vean perturbados irreversiblemente los equilibrios socioecológicos y el patrimonio natural y cultural que albergan (Mata, 2006:4). A esto, se añade una serie de dependencias respecto al continente marcadas por la insularidad. En primera instancia la dependencia político- administrativa al continente, así como de los recursos básicos 
(como combustibles, alimentos, otros bienes y servicios...). Y, por otro lado, la dependencia de flujos de turistas que dinamizan la economía local, aunque con bajo impacto económico y social.

Bajo este contexto y estas dinámicas poblacionales y turísticas, cada vez más crecientes, es importante analizar los flujos estratégicos desde el continente ecuatoriano hacia el archipiélago que sustentan las actividades de turismo en las islas Galápagos. El archipiélago requiere de los recursos necesarios para dar cabida a más de 218.365 visitantes al año (Observatorio del Turismo, 2017), así como a sus 25.124 residentes (INEC, 2015). El territorio necesita producir estas materias, bienes y servicios o, en caso contrario, debido a la insularidad, debe importarlos. Así mismo, este consumo generará residuos que debe asimilar o exportar nuevamente al continente. Los flujos dentro del metabolismo insular siempre serán de doble vía: consumo-residuos. Sin embargo, si poco se ha hecho por estudiar los flujos que ingresan desde el Ecuador continental a Galápagos, mucho menos se han estudiado aquellos flujos de retorno ${ }^{4}$ para determinar su metabolismo insular, sus niveles de vulnerabilidad y viabilidad insular. A esto se suma las restricciones de un territorio natural protegido en donde la conservación de la naturaleza es prioridad y en donde las actividades humanas están limitadas. A pesar de ello, este estudio es un primer aporte, no solo de la necesidad de generar información primaria y un análisis de flujos estratégicos sino más bien desde un abordaje integral de un metabolismo insular y sus capacidades de desarrollo. Aquí recae la necesidad cada vez mayor de generación de estudios sociales y del territorio en Galápagos, así como el uso de la planificación territorial como herramienta para la toma de decisiones.

\subsection{Flujos estratégicos hacia las Islas Galápagos}

En el caso de las islas Galápagos los flujos que garantizan el desarrollo y funcionamiento de las actividades que se desarrollan en las islas se llevan a cabo casi exclusivamente con el Ecuador continental.

Si hablamos de Galápagos como una economía dependiente del turismo existen dos flujos que garantizan que las actividades se desarrollen con normalidad. Estos flujos son: abastos y combustibles (los datos se pueden consultar en el apartado 4).

Los abastos son definidos como mercancías transportadas entre el continente y las islas, e incluyen productos de primera necesidad, materiales de construcción, tanques de gas y otros productos 5 . Su ingreso desde el continente está regulado por SESA-

\footnotetext{
${ }^{4}$ Ver Rodríguez (2014), Senplades (2014).

${ }^{5}$ Las categorías están de acuerdo a la información existente y han sido definidas por el Ministerio de Transporte y Obras públicas a través de los diferentes Puertos del país. En este caso el puerto desde el que se realiza este intercambio se denomina Stereocean y se localiza en la ciudad de Guayaquil.
} 
SICGAL $^{6}$ institución a cargo de evitar la introducción de especies exóticas en el Archipiélago.

Los combustibles, diesel y gasolina, motores clave para el transporte a nivel marino y terrestre en las islas. Son a la vez base del turismo y la movilidad interinsular, y generadores de energía térmica que garantiza el consumo de energía eléctrica a nivel doméstico e industrial.

\subsection{Movilización y transporte entre el Ecuador continental e insular}

Para garantizar el desarrollo de actividades en el archipiélago, así como los servicios turísticos, actividad de la que viven las islas. Es indispensable contar con infraestructura marítima y aérea por donde se realicen los flujos estratégicos que garantizan su metabolismo.

Las islas Galápagos para el desarrollo de sus actividades cuentan con infraestructura en las cuatro islas habitadas y Baltra ${ }^{7}$. Las mismas, permiten el arribo de bienes y servicios por vía marítima y terrestre, y de personas tan solo por vía aérea. Considerando que un avión comercial tarda dos horas en llegar desde el continente (desde Guayaquil) y un barco de carga aproximadamente tres semanas en su arribo. Así, el primer aeropuerto fue el de Isla Baltra construido en el año 1942 con motivos de vigilancia. Ya en 1974 se inauguró el segundo aeropuerto, en la Isla San Cristóbal, anteriormente las personas y los bienes se desplazaban solo mediante barco.

Actualmente (2016) existen tres aerolíneas comerciales que viajan semanalmente al archipiélago y llegan a los aeropuertos de San Cristóbal y Baltra: Aerogal (37\%), LAN (37\%) y Tame (26\%). El puerto aéreo de entrada principal es la isla de Baltra con un 79\% de arribos y la isla de San Cristóbal recibe el 21\% restante (Observatorio de turismo de Galápagos, 2016).

La conexión con las otras islas se realiza mediante desplazamientos inter-islas en lancha o aviones internos. Existen líneas comerciales de pasajeros y limitadísimos charters. Los barcos son de orígenes privados y transportan mercancías desde el continente. También hay barcos y aviones de las Fuerzas Armadas y la Marina que se encargan de cumplir con la misión logística entre las islas y el continente. Los puertos en cada isla, sin embargo, son insuficientes en capacidad y no existe un sistema de almacenamiento de la carga en ninguna con lo que la vulnerabilidad para el sistema alimentario creciente.

\footnotetext{
${ }^{6}$ El Reglamento de Control Total de Especies Introducidas de la Provincia de Galápagos, publicado en el Registro Oficial No. 3516 Segunda edición del 31 de marzo del 2003. Determina los aspectos importantes para la sanidad agropecuaria de Galápagos, entre ellos los elementos que pueden o no ingresar en las islas.

${ }^{7}$ Pequeña isla localizada al norte de la isla Santa Cruz, en el 2010 la isla de Baltra albergaba una población flotante de 14 personas, y actualmente es sede del principal aeropuerto del archipiélago cuyos flujos de turismo se redistribuyen desde Santa Cruz a otras islas. Baltra alberga el aeropuerto del Municipio de Santa Cruz y alberga una zona de almacenamiento de combustible.
} 


\subsection{Problemática para determinar el metabolismo insular}

La información sobre composición, volumen, peso y distribución de la carga asociada a la vía aérea es poco conocida. En relación con la carga por vía marítima solo se conocen porcentajes generales de algunos estudios ${ }^{8}$. Es tan solo a partir del año 2015 que el CREGG se preocupa por determinar la cantidad de toneladas que ingresaban desde el continente ecuatoriano a las islas, estableciendo un cronograma e itinerario de las naves que realizan cabotaje y ruta en las islas. Sin embargo, todos estos itinerarios no contemplan ninguna estrategia o plan global para garantizar el metabolismo social. La situación se agrava ante la limitada capacidad de los puertos para albergar la demanda creciente.

Otro limitante radica en la temporalidad de la información, además de que se encuentra dispersa y solo disponible en carteras de Estado ${ }^{9}$. Por ejemplo, el estudio de Senplades (2014:80) uno de los únicos que analiza el metabolismo en Galápagos para la planificación territorial, menciona que "no se cuenta con suficiente información sobre disponibilidad del recurso hídrico en las islas como para llevar a cabo un balance".

En el caso de los flujos estratégicos, si bien se conocen las rutas realizadas para la distribución de abastos y combustibles entre continente-islas, se desconocen los flujos de carga de la distribución inter-isla, lo que acrecienta la incertidumbre de contabilidad de flujos. La disponibilidad y análisis de los datos efectivos mostrarían las necesidades reales de la población y permitirían plantear estratégicas de ingreso, consumo y salida (residuos) de los bienes necesarios para el vivir cotidiano del territorio Galapagueño y así garantizar una adecuada calidad de vida.

\section{Más allá de los datos: impactos y consecuencias de la dependencia}

\subsection{Consideraciones con relación a la actividad turística}

El crecimiento del turismo en las islas Galápagos es mayor que el del Ecuador continental e incluso que el de Sudamérica en general. Para cubrir este crecimiento entre 1991 y 2006, la tasa de crecimiento anual de la oferta hotelera fue de aproximadamente el 4.8\%. El número total de hoteles en Galápagos incrementó en un $97 \%$ y el número de plazas en un 90\%. Así mismo, desde el 2001 hasta el 2006, el número de vuelos comerciales hacia Galápagos aumentó en un 193\% (Epler y Proaño, 2007).

\footnotetext{
${ }^{8}$ Los conocidos son los estudios de Rodriguez (1993), Zapata (2005), Chiriboga et al. (2006a, 2006b), Proaño (2007), Rodríguez (2014), Senplades (2014), Pizzitutti, F. Mena, C. Walsh, S. (2014).

${ }^{9}$ Se conocen los flujos de abastos a través de información del Ministerio Transporte y Obras Publicas a través de la Subsecretaría de Puertos y Transporte Marítimo y Fluvial. E información sobre cantidades de combustibles por medio de la Empresa Pública de Hidrocarburos, PetroEcuador.
} 
Las políticas en el archipiélago son restrictivas para su crecimiento poblacional endógeno (de cierto modo), sin embargo, no lo son, en cuanto a exigir limitantes o mucho menos disminuir la capacidad de carga de los flujos de turistas. En primera instancia el tipo de experiencia turística ofrecida en las islas Galápagos, turismo de crucero y turismo en tierra serían el origen de este interrogante. Si bien, como lo mencionan Pizzitutti et al. (2014), en los últimos 20-30 años, el turismo de barco dominaba, actualmente el turismo en tierra es mayor. Ello hace pensar que cuanto mayor es el flujo de turismo en tierra, mayores son las necesidades de urbanización y dotación de servicios en la zona de asentamientos humanos, aspecto que ratifican Pizzitutti et al. (2014) quienes mencionan que el turismo en tierra tendrá siempre mayor impacto que el turismo de crucero:

La diferencia entre los turistas basados en barco y la tierra es muy relevante desde el punto de vista del impacto en lo social, terrestres y sub-sistemas marinos. El turismo terrestre implica un mayor desarrollo urbano e infraestructura de apoyo, uso del transporte público y vehículos privados, aumento de la frecuencia de la introducción de especies exóticas, como consecuencia de la importación de la mayoría de alimentos y productos de la tierra firme, el agotamiento de las reservas de agua potable, el consumo de energía, generación de residuos, y las necesidades de un agua mejorada y sistemas de saneamiento en todo el archipiélago.

(Pizzitutti et al. 2014)

Sin embargo, tras las dinámicas que mencionan Pizzitutti et al. (2014) son necesarias además otras capacidades para generarlos, es decir, mano de obra que produzca todos estos bienes y servicios que demanda el crecimiento de la población local y turista. Al no limitar este crecimiento se estaría alterando el metabolismo insular. Por todo ello, además de los impactos causados en los ecosistemas, existen también dinámicas económicas que no garantizan la reinversión de las ganancias económicas en el territorio. Mientras los ingresos por turismo en el año 2006 fueron de 156 millones de dólares directamente en el territorio, los flujos externos fueron de 263 millones, es decir un 169\% mayores. Los estudios más recientes sobre el tema sugieren que tan solo el $15,5 \%$ del valor total del turismo se queda en el archipiélago ${ }^{10}$. Lo que plantea un evidente desequilibrio de flujos económicos e ingresos a pesar de ser el turismo la principal fuente de ellos.

Una revisión de los datos disponibles del Parque Nacional Galápagos muestra que las llegadas de turistas en Galápagos crecieron exponencialmente desde 1995 hasta 2011, con una tasa de crecimiento anual promedio de aproximadamente el $9 \%$ (Pizzitutti et al. 2014). Otro cuestionamiento que compromete la dimensión ambiental

\footnotetext{
${ }^{10}$ Epler et al., 2007.
} 
en las islas Galápagos en relación con la sobrecarga turística. A pesar de que el Plan de Manejo de Galápagos contempla un límite de carga por sector de visita diario, todavía no existe un límite fijo de turistas que deberían ingresar por año a todo el archipiélago.

\subsection{Consideraciones respecto a los combustibles, contaminación ambiental y cambio climático}

Uno de los factores que denotan en mayor medida la vulnerabilidad, en la alta dependencia entre las islas y el continente, es el flujo de combustibles. Tan solo el 6\% de la energía generada en las islas es de origen renovable mientras el resto proviene de la energía térmica generada por combustibles fósiles. Según MICSE (2013) en el año 2012 se importaron en Galápagos 17 millones de barriles de diesel (SENPLADES, 2014:145). El 94\% de la electricidad generada en Galápagos usa como fuente primaria diesel, cuya mayor concentración se encuentra en la isla Santa Cruz con el $62 \%$ del total. El 55\% del consumo de combustibles de Galápagos se concentra en las actividades turísticas y comerciales y el $22 \%$ en la generación de electricidad (SENPLADES, 2014).

Más allá de la dependencia, esta dinámica de flujos le significa al archipiélago una problemática medioambiental compleja que puede afectar las características de sus ecosistemas, como es la generación de gases de efecto invernadero por utilización de energía contaminante, problemas de contaminación de agua por trasporte de materiales desde el continente hacia las islas e inter-islas y la generación de residuos.

La problemática asociada al transporte de combustibles debería ser parte las competencias directas de la planificación ya que de su gestión depende que se pueda garantizar la conservación de los ecosistemas. Así, a raíz de un incidente grave en 2001, producido por el encallamiento del Buque Jessica en costas de la isla San Cristóbal, el peligro real de contaminación ambiental se evidenciaba y obligaba a tomar medidas: "Luego de que un buque-tanquero derramara 240.000 galones de combustible en las aguas alrededor de las islas en el 2001, WWF y Petroecuador, empresa petrolera estatal ecuatoriana, identificaron el transporte de combustible y su almacenamiento como un asunto ambiental importante que necesitaba ser abordado inmediatamente" Larrea (2009). A pesar de ello, nuevos accidentes ocurrieron en aguas del archipiélago en 2014, 2015 y 2017, confirmando que este problema aún no está resuelto ni recibe la importancia adecuada en cuanto a su planificación y gestión. A todo ello se añade que tan solo desde el año 2016 hay un esfuerzo del gobierno regional por conocer la carga de los buques que ingresan en el archipiélago. Sin embargo, aún están en desconocimiento los detalles de composición de carga y los datos de los ingresos por vía aérea, lo que aumenta el riesgo por contaminación de sustancias desconocidas.

Por otro lado, en Galápagos tan solo el $6 \%$ de energías son renovables de origen solar y eólica. La debilidad de las energías renovables radica en la necesidad de mayor espacio físico, al respecto SENPLADES (2014) menciona que el sistema eólico requiere 32 veces más terreno para producir lo mismo que el térmico. Además, 
SENPLADES (2014) menciona que Galápagos es dependiente neto de energía transportada desde el continente, principalmente de diesel, gasolina, GLP, Jet fuel y AVGAS; y esto es evidencia de que Galápagos se encuentra lejos de alcanzar la suficiencia energética deseada.

\subsection{Consideraciones en relación a los abastos}

Si se analizan brevemente los porcentajes que constituyen los abastos llama mucho la atención que casi el $50 \%$ de todo lo que se transporta desde el continente hacia Galápagos lo constituyen los materiales de construcción ${ }^{11}$. A pesar de ello, el resultado es muy sugerente ante el crecimiento poblacional, la migración relacionada con la necesidad de mano de obra y el posible boom turístico.

Mientras la población crece a un ritmo acelerado, los flujos del turismo aumentan, así como la demanda de bienes y servicios por lo que el sector hotelero también necesita ampliar su capacidad de alojamiento. Si actualmente, como hemos visto, el turismo de tierra es mayor al turismo de crucero ello significa para el archipiélago una demanda cada vez mayor de materiales para la construcción de la nueva infraestructura necesaria para albergar esta demanda.

El impacto del volumen de turistas sobre la estructura local es muy amplio, por esto la capacidad de carga turística no solamente subyace dentro de "el número de personas que ingresan en un año", sino también en todas las consecuencias que trae consigo una mayor demanda de nuevos servicios de alojamiento, de alimentación, entretenimiento y otros servicios en general para los turistas. Tras cada uno de ellos, se encierra la necesidad de una infraestructura adecuada que le dé soporte y, en consecuencia, materiales de construcción llevados desde el continente para construirlos, mano de obra capaz de construir esta infraestructura, y más aún, migración de población desde el continente hacia las islas. Toda esta cadena de bienes, productos y servicios para garantizar la economía local dependiente del turismo, genera entonces impactos en el territorio, aún mayores que los tradicionalmente analizados en procesos de boom turístico tradicionales dentro del continuo continental de cualquier destino.

Por otra parte, el desconocimiento de la oferta y demanda de productos alimentarios y de primera necesidad es otro de los elementos claves de la estrategia a seguir. Los productos de primera necesidad representan el 33\% del total de los suministros transportados desde el continente ${ }^{12}$. En cuanto a los alimentos que importa el sector turístico para su consumo, el $80 \%$ llega por vía aérea y en su mayoría $(80 \%)$ son productos orgánicos frescos, como vegetales, frutas, verduras, lácteos y embutidos (Proaño, 2007).

\footnotetext{
${ }^{11}$ Senplades (2014) menciona al cemento, asfalto, ferretería, cerámica/mármol y hierro como los materiales transportados con mayor volumen. El Cemento con diferencia 10 a 1 con relación al resto de materiales.

${ }^{12}$ El Ministerio de Transporte y Obras Publicas menciona un número de 49 productos catalogados de primera necesidad.
} 
Más allá de estos datos, en general existe un gran desconocimiento de la oferta y la demanda de productos alimenticios en el archipiélago. Así, en el caso de la elaboración de productos locales, no existen datos fiables de la carga general que suponen, lo que se traduce en la necesidad urgente de generación de dicha información. Ante el desconocimiento de la oferta y la demanda no se pueden generar alternativas que reduzcan la dependencia del continente y mucho menos la generación de inversión tecnológica y/o agropecuaria que mejore los productos locales sin atentar contra el bienestar de los ecosistemas endémicos.

Mientras tanto, la llegada de alimentos desde el continente sigue aumentando para satisfacer la creciente demanda de la población residente y visitante. Los productores agrícolas que aún se dedican a esta actividad en Galápagos, por su parte, no cuentan con políticas claras de desarrollo y se enfrentan a costes de producción muy altos. La mano de obra es cara y escasa, los insumos no son fáciles de conseguir, ya que deben ser llevados des del continente, y frecuentemente no son los adecuados, pues hay restricciones severas en cuanto al tipo de abono, fertilizantes y -por supuestoplaguicidas y herbicidas que se pueden utilizar (Proaño, 2007:80).

Desde el año 2013 el Plan de Bioagricultura de Galápagos, desarrollado como estrategia por el Ministerio de Agricultura del Ecuador, tenía el objetivo de "contribuir a la soberanía alimentaria de la población local y disminuir la incidencia de las especies vegetales invasoras en el área agropecuaria" (Guzmán y Poma, 2015). A pesar de ello, Senplades (2014:43) menciona que apenas el 15,31\% de las tierras se encuentran utilizadas de acuerdo con sus aptitudes biofísicas, lo que significa que el $84,69 \%$ de las tierras se encuentran mal utilizadas. Paralelamente, según normativa, el espacio destinado para los asentamientos humanos debería ser el 3,3\% del archipiélago, sin embargo, Senplades (2014:49) menciona que actualmente el 3,76\% está siendo destinado a ese uso. Esto significa que al menos $3.787,11$ hectáreas de suelo están en conflicto de uso. Ante estas situaciones de dependencia, pero al mismo tiempo de falta de posibilidad de generar estrategias de planificación a seguir, el gobierno ecuatoriano, a través de diversos planes de desarrollo como el buen vivir, sigue realizando incentivos económicos en las islas.

\subsection{Incentivos económicos y políticas contradictorias en las islas Galápagos}

A pesar de los intentos de conservación-desarrollo de las islas, algunas políticas se evidencian contradictorias con su objetivo final: alcanzar el buen vivir en Galápagos. Principalmente centradas en restringir los flujos desde el exterior, dichas iniciativas no se preocupan especialmente en analizar las dinámicas y el funcionamiento del territorio en su totalidad. En la actualidad se continúa trabajando con políticas de insularidad establecidas hace varias décadas cuando aún se pretendía la colonización de las islas, siendo que actualmente hay un crecimiento poblacional acelerado paralelo al crecimiento desproporcionado del turismo.

La contradicción entre los instrumentos, estrategias, planes de desarrollo y políticas territoriales existentes son evidentes en numerosos ámbitos, un ejemplo de 
ello es el principio número 7 de la LOREG (2015) en referencia al derecho al acceso preferente: "Las personas residentes permanentes de la provincia de Galápagos tendrán que ser consideradas de manera preferente... Asimismo, gozarán de derecho preferente en el acceso a recursos naturales y a las actividades ambientalmente sostenibles que se lleven a cabo en dicha provincia ${ }^{13}$. Así, diversos incentivos económicos y socioambientales, como éste, impulsan la migración desde el continente hacia Galápagos y paradójicamente hacen que la región sea mucho más atractiva para vivir que otras del territorio ecuatoriano continental.

\subsubsection{Beneficios en transporte y ayudas salariales}

Un ejemplo en relación al transporte se encuentra en la Disposición General tercera sobre tarifas de transporte aéreo y marítimo, donde se estipula, como en numerosos territorios insulares de otros estados, que para los residentes permanentes y temporales, las tarifas de transporte aéreo y marítimo de pasajeros tienen preferencias "El Estado ecuatoriano, a través del órgano competente de la rectoría del transporte, determinará la o las tarifas preferenciales para el transporte aéreo de pasajeros y carga de residentes permanentes y temporales de la provincia de Galápagos (...) que se trasladen en un servicio entre islas o desde las islas hacia el Ecuador continental o viceversa" (LOREG, 2015).

Según la LOREG (2015), en las Disposición Generales Cuarta en referencia a la actualización del régimen salarial para el sector publico menciona: "Las remuneraciones de los trabajadores del sector público y privado de la provincia de Galápagos se pagarán con un incremento que se calculará multiplicando el índice de precios anual al consumidor con respecto a los precios del Ecuador continental. Esta disposición se aplicará a todos los trabajadores públicos y privados (...).

\subsubsection{Subsidios y ayudas en el sector energético}

De igual modo, en Ecuador las ayudas han sido y son una política de Estado y están estipuladas de manera preferencial en el caso de la provincia de Galápagos. En los últimos años, la situación geográfica de las islas y la posición del Gobierno del Ecuador por mantener su política de subsidios en el sector energético, se han traducido en una alta contribución de recursos estatales hacia esta provincia. Además, la ubicación del archipiélago ha originado que muchas actividades se gestionen en las islas considerándolas como un sistema aislado del Ecuador continental (Jacome, 2007). A nivel general, en el Ecuador en el año 2013 los subsidios representaron $6.603,68$ millones de dólares, siendo casi el $69 \%$ de este valor destinado a los

\footnotetext{
${ }^{13}$ En referencia al artículo n. 3 de la LOREG 2015. Título I, disposiciones preliminares, Capítulo I: Objeto, ámbito, finalidades y principios de esta Ley. Principio número 7.
} 
subsidios de combustibles. ${ }^{14}$ Dentro de estos subsidios y ayudas, es interesante pararse a analizar los valores asumidos para los combustibles y la generación de energía por parte del Gobierno nacional del Ecuador en las islas Galápagos ${ }^{15}$

Así, en el caso de las ayudas para el abastecimiento de combustibles fósiles, el subsidio equivale a la diferencia entre el costo de los derivados y al precio de venta en las islas. Al respecto Jacome (2007) menciona que para el año 2005 la población de Galápagos necesitó de 9.405.048 de galones, lo que le supuso al estado un valor por subvenciones de 13.402.294 dólares. Los valores del subsidio por combustible fueron los siguientes: de US\$ 0,92 por galón de diesel empleado en la generación térmica, US\$ 1,01/gal para el diesel empleado en el transporte y US\$1,48/gal para la gasolina extra. El valor asignado para subsidiar la demanda de combustibles empleados en el sector de transporte fue de US\$10,48 millones, lo que representa el 78\% del total de las ayudas por combustibles fósiles. Si para el año 2015 la demanda de combustibles ha incrementado a 17 millones, sin duda el coste de las ayudas sigue en aumento y, según fuentes recientes, del total de subsidios a los combustibles, el $90 \%$ es absorbido por las actividades turísticas (Senplades, 2014).

Otro aspecto subvencionado desde el estado es el sector eléctrico. La generación de electricidad en el archipiélago se realiza en centrales térmicas que son más costosas que las centrales hidroeléctricas utilizadas en el sistema nacional interconectado. Sin embargo, como menciona Jacome (2007) los precios de la electricidad en las islas no difieren sustancialmente de los precios de la electricidad en el Ecuador continental. Por lo que el subsidio sería aún mayor en el archipiélago.

En definitiva, según menciona Senplades en 2014, el Gobierno central envía a las islas USD \$ 98,2 millones anuales en forma de subsidios energéticos, salarios del sector público, transferencias a los Gobiernos Autónomos Descentralizados e inversión pública.

En términos económicos existen tres factores que requieren una urgente revisión por parte de las autoridades del Estado. Por un lado, la redistribución de la renta; por el otro, la captura del flujo de divisas no territorializado; y finalmente, la absorción de un importante porcentaje de los subsidios estatales por parte del turismo (Senplades, 2014:264).

\footnotetext{
${ }^{14}$ Diario El Universo, 2013.

${ }^{15}$ En base a Jácome (2007) para determinar el subsidio de los derivados de petróleo en el Archipiélago de Galápagos estableció varios criterios. Primero, el costo del diesel y la gasolina extra en cada isla. Considerando el precio del derivado de petróleo como producto importado (costo de oportunidad). A estos costos le agregó, los costos correspondientes al flete marítimo desde el continente hacia las islas y al transporte terrestre desde el muelle al sitio de almacenamiento de combustibles, así como el impuesto al valor agregado IVA y los gastos de comercialización.
} 


\section{A modo de conclusiones}

Con la información expuesta a lo largo del análisis se evidencia que el alto nivel de dependencia e interdependencia territorial que tienen las Islas Galápagos con el Ecuador continental probablemente no sea mayor que en el caso de otras islas, pero se encuentra potenciado por los limitantes de uso de recursos y la política de conservación del área natural protegida. En general se aprecia que existe un desconocimiento de las dinámicas y flujos entre el continente y las islas Galápagos. La información está dispersa y centrada en instituciones a nivel nacional y hay pocos esfuerzos por recopilar, diagnosticar y analizar los flujos particulares más allá de los turísticos. Por otro lado, los estudios tienen un sesgo ambiental y no están insertos en un contexto de planificación territorial y social.

Desafortunadamente la gestión y manejo de las áreas de asentamientos humanos versus el área protegida han sido relegados a un segundo plano. Las áreas bajo conservación son de las cuales se aprovecha el turismo y en muchos casos, miles de turistas desconocen que existen seres humanos que también comparten este hábitat. Si bien, la mayoría de productos, bienes y servicios de desplazan en dirección continente-islas (propia de la insularidad), estos flujos no se dan con equidad territorial, es decir, no inciden económica, ambiental, física y temporalmente de la misma forma. Los impactos positivos y negativos afectan en ambos territorios y la alteración de uno de estos flujos incidirá en su funcionamiento, pero no del mismo modo ni con la misma intensidad. De esta forma la variación de la dinámica significa un nivel de vulnerabilidad para el territorio galapagueño y por ello la importancia de conocer la forma en que se generan e inciden estos flujos.

En el caso de las islas Galápagos, el análisis de la insularidad y del metabolismo insular, desde la perspectiva geográfica con énfasis en las diversas perspectivas del desarrollo, se vuelve cada vez más necesario y adquiere importancia al incluir el componente geográfico como instrumento de espacialización de las realidades del territorio. Los flujos de intercambio de entrada (recursos) y salida (residuos) del territorio galapagueño son claves para la planificación prospectiva. Parte de la sostenibilidad radica en garantizar a las generaciones futuras recursos. Pero entonces, cómo garantizarlos si aún se desconoce que huella se deja tras el paso y el uso del territorio galapagueño.

La planificación y las políticas públicas se han adaptado a la realidad insular galapagueña, principal y casi exclusivamente en el ámbito económico y de finanzas públicas. Los recursos económicos representan el 100\% adicional en relación con el resto de territorios del Ecuador continental, además de los diversos subsidios que en este contexto reciben los residentes, como es el caso de las ayudas a la gasolina, los transportes aéreos, la electricidad, entre otros.

Las políticas públicas en Galápagos se han centrado en la conservación de los recursos naturales, dinámica lógica al ser un área protegida, pero parecen haberse olvidado de que en las islas habitan seres humanos que tienen necesidades como las de cualquier otro habitante del continente. La población de las Islas Galápagos, a pesar de gozar de la suerte de vivir en un ambiente de paz único en el mundo, está 
limitada por una serie de restricciones consecuencia de su consideración de área protegida. Sin embargo, el gobierno regional, como autoridad rectora, es el responsable de solventar y dar respuesta a cada una de ellas, pensando que estos recursos deben ser generados desde el territorio galapagueño o transportados desde el exterior.

Entonces, ¿se planifica realmente en las áreas de asentamientos humanos? ¿Se ha considerado los impactos que las actividades humanas están dejando en el resto del territorio insular? ¿Cómo reducir la vulnerabilidad generada por estas dependencias con el continente? Al realizar este estudio se han encontrado variables que nos hablan por sí solas y muestran como la falta de una planificación real permite el desarrollo de un turismo masivo y la balearización (Blázquez et al., 2011) de estos espacios turísticos insulares al no ponen límites y privilegiando los beneficios económicos por encima de las consecuencias directas sobre la población local, el territorio e incluso el medioambiente.

Ejemplo de la necesidad de una planificación adecuada es el desconocimiento exacto de los flujos de suministros y por lo tanto de las necesidades de la población, del tipo de consumos que se realizan: de qué se alimentan los galapagueños y los turistas ¿Qué se puede producir localmente y qué no? ¿Cuáles son los materiales de construcción utilizados? ¿Pueden éstos atentar al medioambiente? ¿En qué porcentaje se usan? ¿Cómo se construye en Galápagos? Los datos de abastos nos dicen que el $50 \%$ del total de productos llegados a las islas son materiales de construcción, esto indica la magnitud imparable de crecimiento exponencial, ¿Cuánto más se espera crecer? ¿En qué isla se construye más?

En el caso de la dependencia de abastos específicamente de alimentos, si no se fortalecen las políticas de producción agropecuaria local la dependencia al continente seguirá en aumento. Paralelamente, mientras no mejoran las condiciones de vida de los habitantes de las zonas rurales, esta población se verá atraída por actividades de mucho mayor rédito económico como lo son las actividades asociadas al turismo, dejando los pocos espacios cultivados en abandono y haciendo cada vez más necesaria la mano de obra llegada desde el exterior.

Por otro lado, a pesar de que el Gobierno regional impone medidas restrictivas en algunos ámbitos, las políticas de desarrollo regional, en muchos casos, eximen de las mismas a su población local. Estas políticas, enmarcadas dentro del desarrollo de régimen especial se convierten en incentivos económicos que convocan nuevamente a la migración desde el exterior, así como en muchos casos gestan un sistema de baja competitividad local.

En relación con los combustibles, generación de energía y transporte, debido a la insularidad los subsidios significan un montante más alto que para el resto de ecuatorianos del continente. Por ello, en este caso, la apuesta por energías alternativas en Galápagos es clave y varios proyectos siguen en marcha en las islas pobladas.

En cuanto a las políticas y normativa adaptadas a la realidad insular se plantea un interrogante clave en relación con su posible permisividad con el tratamiento preferente a la población local en comparación con el nivel de restricción impuesta a 
la población del continente. En Galápagos, el aumento del ingreso de turistas no es la única problemática existente, sino también un crecimiento poblacional sin límites.

En definitiva, existe un sinfín de estudios ambientales y de biodiversidad en las islas, sin embargo, quedan muchos interrogantes por responder y es necesario plantear estudios globales que imbriquen el análisis territorial y social para poner en evidencia las diversas realidades del territorio y su complejidad. Las especies naturales en este maravilloso y biodiverso paraíso son claves, pero la población que habita en las islas también. El conocimiento de su crecimiento y sus demandas serán las únicas que garantizarán que este gran ambiente de coexistencia pueda ser sostenible o no. Además, el abordaje de la planificación territorial del desarrollo como una herramienta, planteando los espacios naturales y de asentamiento humanos como un sistema territorial complejo. Todas estas propuestas son imprescindibles en una reflexión profunda, y necesarias para considerar las necesidades reales de las poblacionales en la toma de decisiones.

El enfoque de metabolismo insular permite ahondar en el estudio de espacios complejos. Considerando al archipiélago como sistema de islas interdependientes unas con otras y no como se ha considerado tradicionalmente a cada isla como sistema independiente. Así mismo el abordaje como socio-ecosistema o sistema complejo permite conocer además este territorio sobre el cual las dinámicas poblacionales y turísticas deben apuntar el desarrollo de políticas públicas de planificación para garantizar como tal la viabilidad insular.

Contradictoriamente, a pesar de la falta de planificación, el sistema socioecológico, por sus constantes cambios y problemáticas, se acerca más a la resiliencia socioecológica. Terminología cuya aplicabilidad en el caso Galápagos está todavía por analizar.

\section{Bibliografía}

Baldacchino, G. (2004): The coming of age of island studies. Tijdschrift voor Economische en Sociale Geografie, 95(3), 272-283.

Barceló, B. (1985): Introducció a la nisologia. A À. d. Barcelona (Ed.), I Conferència Econòmica de la Mediterrrània Nord-Occidental. (p. 2071). Barcelona: Fundació del Congrés de Cultura Catalana.

Barceló, B. (1997): Illes, Illeïtat i Insularitat. Les Illes Balears, per exemple. Comunicacions dels membres de la Secció de Filosofia i Ciències Socials XXII (p. 111-127). Barcelona: Institut d'Estudis Catalans.

Boadas, A. (2011): Islas e insularidad geográfica. Revista Terra Nueva Etapa 2011 XXVII (41). Págs. 149-155. Red de Revistas Científicas de América Latina, el Caribe, España y Portugal. Universidad Autónoma del Estado de México Revisado online. Mayo 2016 en http://www.redalyc.org/pdf/721/72119117007.pdf

Blázquez, M.; Murray, I.; Artigues, A. (2011): La balearización global. El capital turístico en la minoración e instrumentación del Estado. Investigaciones Turísticas. $\mathrm{N}^{\circ} 2$, juliodiciembre, 2011, pp. 01-28. 
Chiriboga, R. Maygan, S. Fonseca, B. (2006a): Caracterización de los sistemas de producción en Galápagos en relación con el fenómeno de las Especies Invasoras. Producto 1. Proyecto ECU/00/G31“Especies invasoras de las Galápagos”. GEF. Ministerio del Ambiente del Ecuador. INGALA. PNUD. 121 Págs. Puerto Ayora, Galápagos, Ecuador.

Chiriboga, R. Maygan, S. Fonseca, B. (2006b): Zonificación agroecológica de las zonas agropecuarias en relación con las especies invasoras. Producto 2. Proyecto ECU/00/G31“Especies invasoras de las Galápagos”. GEF. Ministerio del Ambiente del Ecuador. INGALA. PNUD. 86 págs. Puerto Ayora, Galápagos, Ecuador.

DPNG (2005): Plan de Manejo Parque Nacional Galápagos. Dirección del Parque Nacional Galápagos. AECID. USAID. BID Revisado en línea. Septiembre del 2016 en: http://www.galapagospark.org/documentos/DPNG_plan_de_manejo.pdf

DPNG (2014): Informe anual de visitantes a las áreas protegidas de Galápagos 2015. Fundación Charles Darwin, Ministerio del Ambiente. Revisado en línea. Octubre 2016 en: http:/www.galapagos.gob.ec/wp-content/uploads/downloads/2016/08/Informe-deVisitantes-2015.pdf

DPNG, CGREG, FCD y GC. (2013): Informe Galápagos 2011-2012. Puerto Ayora.

Epler, B. (2007): Tourism, the economy, population growth, and conservation in Galápagos. Fundación Charles Darwin. Puerto Ayora, Galápagos, Ecuador.

Epler, B. \& Proaño (2007): El turismo en Galápagos: una tendencia al crecimiento. Págs. 3135. En Informe Galápagos 2006-2007. Puerto Ayora, Galápagos, Ecuador.

Epler, B. Watkins, G. y Cardenas, S. (2007): Los flujos financieros del turismo de Galápagos. En Informe Galápagos 2006-2007. Puerto Ayora, Galápagos, Ecuador.

Escalera Reyes, J. \& Ruiz Ballesteros, E. (2011). Resiliencia Socioecológica: aportaciones y retos desde la Antropología. Revista de Antropología Social, 20, 109-135.

EUROSTAT (2012): Economy-wide material flow accounts (EW-MFA). Compilation Guide 2012. Luxembourg: European Commission.

http:/ec.europa.eu/eurostat/documents/1798247/6191533/2013-EW-MFA-Guide10Sep2013.pdf/54087dfb-1fb0-40f2-b1e4-64ed22ae3f4c

Fischer-Kowalski, M. and H. Haberl (eds.) (2007): Socioecological transitions and global change: trajectories of social metabolism and land use. Cheltenham: Edward Elgar.

Fischer-Kowalski, M., F. Krausmann, S. Giljum, S. Lutter, A. Mayer, S. Bringezu, Y. Moriguchi, H. Schütz, H. Schandl and H. Weisz. (2011): Methodology and indicators of economy-wide material flow accounting. Journal of Industrial Ecology 15 (6): 855-875. http://onlinelibrary.wiley.com/doi/10.1111/j.1530-9290.2011.00366.x/abstract

Godenau, D. \& Hernandez, R. (1996): Insularidad ¿un concepto de relevancia analítica? Universidad de La Laguna. Estudios Regionales N45. Págs. 177-192. Canarias, España.

Guzman JC \& JE Poma. (2015): Bioagricultura: Una oportunidad para el buen vivir insular. Pp. 25-29. En: Informe Galápagos 2013-2014. DPNG, CGREG, FCD y GC. Puerto Ayora, Galápagos, Ecuador.

Hepburn, E. (2010): A comparative analysis of island region autonomy. Canadian Political Science Association Conference. Montreal, Québec.

Hercowitz, M., \& Alier, J. M. (2003): Metabolismo social y turístico de Lanzarote. La Caja Insular de Ahorros de Canarias.

INEC. (2015): Resultados del Censo poblacional 2015 de población y vivienda en Galápagos. 
Jácome. C. (2007): Subsidios en el sector energético insular. En Informe Galápagos 20062007. INGALA. p. 67-72. Santa Cruz, Galápagos, Ecuador.

Larrea, I. (2009): Terminal de Combustibles de Galápagos Obtiene Certificación Ambiental. Boletín Galápagos. WWF. Galápagos, Ecuador.

Ley Orgánica de Régimen Especial de Gobierno de Galápagos. LOREG. Registro Oficial Suplemento 520 de 11-jun.-2015. Quito, Ecuador.

Newell, J.P. and Cousins, J.J. (2014): The boundaries of urban metabolism: towards a political-industrial ecology. Progress in Human Geography. Vol 39, Issue 6, pp. 702-728.

Manera. C. Garau. J. (2005): Los costes de la insularidad en el desarrollo económico. Un contraste en el mediterráneo occidental (1987-2002). Colección Mediterráneo e Historia Económica. $\mathrm{N}^{\mathrm{o}}$ 7. Instituto de Estudios Socioeconómicos de Cajamar. Págs. 247-268Baleares, España. Revisado en línea. Junio 2016 en:

http://www.publicacionescajamar.es/pdf/publicaciones-periodicas/mediterraneoeconomico/7/7-98.pdf

Moles, A. A. (1982): Nissonologie ou science des îles. L'espace Géographique(4), 281-289.

Murray-Mas, I. (2015): The fishing footprint of a tourism-based economy: displacing seafood consumption from local to distant waters in the Balearic Islands. Journal of Political Ecology 22: 211-238.

Observatorio de Galápagos. http://www.observatoriogalapagos.gob.ec/

Pons, A. (2016): Turisme, illeïtat i urbanització a les Illes Balears (1956-2006). Tesis doctoral. Universitat de les Illes Balears.

Pizzitutti, F. Mena, C. Walsh, S. (2014): Modelling tourism in the Galapagos Islands: An Agent-Based Model Approach. Journal of Artificial Societies and Social Simulation. Revisado en línea. Julio 2016 en: http://jasss.soc.surrey.ac.uk/17/1/14.html

Proaño. M. (2007): Evaluación de escenarios de expansión turística en Galápagos. INGALA. Ministerio del Ambiente, PNUD. Fundación Charles Darwin. SICGAL. GEF. Puerto Ayora, Galápagos, Ecuador. Revisado en línea. Julio del 2016 en: http://manejoespeciesinvasoras.info/wiki/images/8/84/6_3_Escenarios_de_Expansion_Turi stica.pdf

Rodríguez. J. (1993): Las islas Galápagos. Estructura geográfica y Propuesta de Gestión territorial. Universidad Católica de Nijmegen, Holanda. FESO. Fundación Charles Darwin. Fundación Natura. JULA/CELCADEL. Ediciones Abya-Yala. Pg. 276. Quito, Ecuador.

Rodríguez, G. (2014): Territorios insulares y dependencia funcional: el caso de las islas Galápagos, Ecuador. Tesis de máster para la obtención del título en Planificación Territorial y Gestión Ambiental. Universidad de Barcelona. Barcelona, España.

Senplades (2014): Informe Técnico Consolidado. Diagnóstico y análisis biofísico para evaluación y formulación de escenarios de desarrollo en el Archipiélago de Galápagos. Informe Técnico CEPROEC IT2014_01 Centro de Prospectiva Estratégica /Instituto de Altos Estudios Nacionales/ Secretaria Nacional de Planificación y Desarrollo. Quito, Ecuador.

Watkins, G. (2008): A paradigm shift in Galapagos research. Galapagos Research, 65, 30-36.

Zapata. F. (2005): Diagnostico. Sistema de transporte de carga hacia y entre las islas. INGALA. Ministerio del Ambiente, PNUD. Fundación Charles Darwin. SICGAL. GEF. Puerto Ayora, Galápagos, Ecuador. 